\title{
Tendencias de mortalidad por cánceres atribuibles al tabaco en México
}

\author{
Víctor José Tovar-G uzmán, MD, MSc, ${ }^{(1,2)}$ Simón Barquera, MD, MSc, ${ }^{(1)}$ \\ Francisco J López-Antuñano, MD, MPH. ${ }^{(1,2)}$
}

\section{Tovar-Guzmán VJ, Barquera S, López-Antuñano FJ. Tendencias de mortalidad por cánceres atribuibles al tabaco en México.}

Salud Publica Mex 2002;44 supl 1:S20-S28.

El texto completo en inglés de este artículo está disponible en: http://www.insp.mx/salud/index.html

\section{Resumen}

Objetivo. D escribir la tendencia de la mortalidad por cáncer atribuible al consumo de tabaco, particularmente neoplasias del pulmón, en México, durante el periodo 1980-1997. Material y métodos Se hizo un análisis de tendencias de mortalidad para cada tipo de cáncer asociado con el tabaco, según la C lasificación Internacional de Enfermedades. Las tasas cruda y ajustada de mortalidad fueron estimadas en términos de edad, género, causa básica de la muerte y año, entre 1980 y 1997. Se estimó la razón de género y proporción relativa para los casos que estaban en el grupo de edad entre 35 y 64 años y para to da la población estudiada. Como denominador se utilizaron las proyecciones por edad calculadas por el Consejo N acional de Población (1970-2010). Resultados. La razón de género para las tasas de mortalidad por cáncer de laringe, esófago, cavidad oral y faringe fue de 2.10:1.00 (hombre: mujer). La razón de género para el cáncer de laringe es enorme: 4.21: 1.00, probablemente debida a la mayor prevalencia de hombres fumadores de tabaco. La proporción relativa estimada, usando la mortalidad total debida a tumores malignos, entre 1980 y 1997 fue la siguiente: cáncer de pulmón $12.31 \%$, laringe $1.71 \%$, esófago $1.55 \%$ y cavidad oral/faringe $1.49 \%$. El antecedente de tabaquismo tiene correlación con la tendencia de la tasa de mortalidad por cáncer del pulmón (Beta: 0.910, IC 95\%: 1.097-1.797; $\left.R^{2} 0.827\right)$. Para los grupos sociales mas pobres, por entidad federativa, la correlación está invertida (Beta: -0.510 , IC 95\%: $\left.-0.170,-0.039, R^{2}: 0.260\right)$. Conclusiones En México, la mejoría en el diagnóstico, la transición demográ-

\author{
Tovar-Guzmán VJ, Barquera S, López-Antuñano FJ. \\ Mortality trends of cancer attributable \\ to tobacco smoking Mexico. \\ Salud Publica Mex 2002;44 suppl 1:S20-S28. \\ The English version of this paper \\ is available at: http://www.insp.mx/salud/index.html
}

\begin{abstract}
A bstract
Objective. To describe the mortality trends of cancer attributable to tobacco smoking, particularly lung cancer, for the 1980-1997 period in Mexico. Material and Methods. Mortality trends were analyzed for each type of cancer associated to tobacco smoking, according to the International Classification of D iseases (ICD ). C rude and adjusted mortality rates were estimated for the period between 1980 and 1997, by age, gender, basic death cause, and year of death. The gender ratio and the relative proportion were estimated for cases in the 35-64 age group and for the entire study population.Age population projections by Consejo $\mathrm{N}$ acional de Poblacion ( $\mathrm{N}$ ational Population Council), were used as denominators (1970-2010). Results. The gender ratio for mortality rates for lung, esophageal, oral cavity and pharyngeal cancer was 2.10:1.00 (male: female). The gender ratio for laryngeal cancer was striking: 4.21:1.00, probably due to the higher prevalence of male tobacco smokers. The estimated relative proportion, using the total mortality due to malignant cancers between 19801997 , was $12.31 \%$ for lung cancer, $1.71 \%$ for larynx cancer, $1.55 \%$ for esophageal cancer, and $1.49 \%$ for oral cavity/pharyngeal cancer. Previous tobacco smoking was correlated with the mortality rate trends for lung cancer (beta: 0.910 , IC 95\%:1.097-1.797, $\left.R^{2} 0.827\right)$. For the poorest social groups by federal entity, the correlation was inverted (beta: -0.510 , IC $\left.95 \%-0.170,-0.039, R^{2}: 0.260\right)$. Conclusions In Mexico, increased tobacco smoking, improved cancer diagnosis, and the demographic transition, are probably the main factors
\end{abstract}

(1) Istituto Nacional de Salud Pública. Cuernavaca, Morelos, México.

(2) Grupo Catalizador de Acciones contra el Tabaquismo, Secretaría de Salud México, D.F., México.

Fecha de recibido: 12 de febrero de 2001 - Fecha de aprobado: 10 de enero de 2002

Solicitud de sobretiros: Dr. Simón Barquera. Centro de Investigación en Salud Poblacional (CISP). Instituto N acional de Salud Pública (IN SP).Avenida Universidad 655, Colonia Santa María A huacatitlán 62508 Cuernavaca, Morelos, México sbarquera@correo.insp.mx 
fica y el incremento del consumo de tabaco son, probablemente, los principales factores a los que se atribuye la tasa de mortalidad por cáncer. N o obstante, otras variables asociadas con el estilo de vida, como urbanización, actividad física, ingesta de caroteno ides y otros componentes dietéticos y tóxicos, como el alcohol, podrían también influenciar las tasas de mortalidad. A pesar de que el cáncer relacionado con el tabaco es un problema de salud pública con un pobre pronóstico, y de que está aumentando dramáticamente, el mayor factor de riesgo (el tabaco) puede eliminarse con educación, promoción de la salud, regulación de la publicidad y políticas fiscales saludables. El texto completo en inglés de este artículo está disponible en: http:// www.insp.mx/salud/index.html

Palabras clave: mortalidad/tendencias; neoplasmas pulmonares; tabaquismo; México determining cancer mortality rates. However, other lifestyle associated variables, such as urbanization, physical activity, carotenoid intake, and other dietary and toxic substances like alcohol, may also influence the morbidity and mortality rates. Although tobacco-related cancer is a fast-growing public health problem having a poor prognosis, tobacco smoking, the main risk factor, could be eliminated by health education and promotion, together with publicity regulation and healthy taxation policies.T he English version of this paper is available at: http://www.insp.mx/salud/ index.html

Key words: mortality/trends; lung neoplasms; smoking; Mexico
E 1 tabaco es un factor de riesgo para la salud que requiere mayor decisión política del Estado Mexicano para eliminarlo. La morbilidad y mortalidad atribuibles al consumo de tabaco aumentan significativamente. Las enfermedades asociadas con el subdesarrollo disminuyen lentamente y continúan siendo prioritarias. El cáncer se puede prevenir si se eliminan factores de riesgo como consumo de tabaco, alcohol y alimentos inadecuados. Las intervenciones de mayor costo-beneficio social son la educación y la promoción de la salud. Alrededor de 500 millones de personas que actualmente fuman, eventualmente morirán de causas asociadas con el tabaquismo.

Para 2030, el tabaco será la primera causa de muerte, con alrededor de 10 millones de muertes anuales. Los hombres tienen una tasa de incidencia de 4 a 6 veces mayor que las mujeres. Las tasas de mortalidad son 10 a 15 veces más elevadas para fumadores crónicos.

En los países en los cuales muchos cánceres están empezando a disminuir, aún se observarán incrementos en las tasas de mortalidad por cáncer de pulmón. Se estima que una de cada 10 personas en el mundo morirá debido al tabaquismo; esta estimación se incrementará a una de cada seis personas en el año 2030, o sea alrededor de 10 millones de muertes por año.

En México, los problemas de salud relacionados con el consumo de tabaco representan un importante reto para los planificadores de la política de salud. La morbilidad y la mortalidad debidas a las enfermedades crónicas no transmisibles (ECNTs) están aumentando significativamente y los daños asociados con el subdesarrollo (como la diarrea y la desnutrición), están decreciendo paulatinamente, pero aún son prioritarios. A este fenómeno se le ha denominado transición epidemiológica; a México se le ha identificado como uno de los países que experimentan la variante demorada del modelo de transición. Una de las características especiales de las ECNTs es la relevante asociación con los estilos de vida (poca actividad física, dietas de calidad pobre, estrés, adicciones, etcétera). A la tercera etapa de la transición, cuando la morbilidad y mortalidad se deben principalmente a las ECNTs, se le ha llamado la etapa de las enfermedades hechas por el hombre. . $^{1-3}$

Algunos tipos de cáncer se consideran enfermedades prevenibles en virtud de que muchos de los factores relacionados con el estilo de vida, como el consumo de tabaco, del alcohol y de algunos alimentos, se han asociado con su incidencia. Los factores determinantes de esos cánceres pueden ser eliminados con intervenciones apropiadas (v.gr., promoción y educación para la salud).., 5

Con los patrones actuales de consumo de tabaco, cerca de 500 millones de personas vivas en la actualidad morirán eventualmente por causas asociadas con el consumo de tabaco. Se estima que para el año 2030, el tabaco será la primera causa de muerte en el mundo, con cerca de 10 millones de muertes al año. Los hombres muestran una incidencia 4 a 6 veces mayor que las mujeres. La tasa de mortalidad es 10 a 15 veces mayor en los fumadores crónicos. En los países en donde la incidencia de la mayoría de cánceres empieza a descender, se observará todavía un aumento en la tendencia de las tasas de mortalidad del cáncer de pulmón. Se estima que una de cada 10 personas en el mundo muere por fumar tabaco. Esta estimación 
aumentará a una en cada seis personas para el año 2030, o sea, cerca de 10 millones de muertes por año. ${ }^{6}$

Durante la década de los 50, se publicaron los primeros estudios de casos y controles en donde se encontró la asociación entre fumar tabaco y cáncer de pulmón. ${ }^{7-10}$ Después, otros estudios encontraron asociación entre consumo de tabaco y cáncer de la cavidad oral, laringe y esófago. Alrededor de 1957, se identificaron algunos componentes de los cigarrillos como promotores tumorales, co-carcinogénicos y carcinogénicos órgano-específicos. ${ }^{9,11}$

En México, el cáncer de pulmón es la primera causa de mortalidad entre las neoplasias malignas ${ }^{12}$ y uno de los problemas más relevantes de salud pública; sin embargo, el consumo de tabaco y sus efectos dañinos en la salud se han investigado precariamente tanto en México como en otros países en desarrollo. ${ }^{13-15}$

El objetivo de este estudio es describir las tendencias de la mortalidad -cruda y estandarizada- por edad del cáncer relacionado con el tabaco; en particular las del cáncer de pulmón, de 1980 a 1997, en el grupo de edad de entre 35 a 64 años, en México. Además, se analizan las razones de género para cada tipo de cáncer, así como la correlación entre el consumo previo de tabaco per capita y las tasas de mortalidad, considerando 27 a 30 años de tiempo de exposición. Finalmente, se presenta la correlación entre la marginalización social y las tasas de mortalidad por cáncer del pulmón.

\section{Material y métodos}

Se llevó a cabo un análisis de tendencias de mortalidad de cada tipo de cáncer asociado con el tabaco, usando información del Registro Nacional de Enfermedades, publicado por el Instituto Nacional de Estadística, Geografía e Informática (INEGI) ${ }_{1}^{16}$ por grupo de edad (0-120 y 35-64 años), sexo, estado (32 entidades), causa básica de muerte y año (1980-1997). Se estimaron las razones de sexos y frecuencias relativas para cada tipo de cáncer asociado con el tabaco: a) cavidad oral y faringe (incluyendo boca, labios, paladar y lengua) de acuerdo con la Clasificación Internacional de Enfermedades-1975, CIE-IX,140-148 ${ }^{17}$ ) Organización Panamericana de la Salud (OPS-1978); b) esófago (CIE-IX, 150); c) laringe (CIE-IX, 161), y d) pulmón (CIEIX, 162).

Como denominador para estimar las tendencias de mortalidad se utilizaron las proyecciones de población (Consejo Nacional de Población -Conapo- ${ }^{18}$ 1970-2010). Para cada cáncer atribuible al tabaco, se calcularon las tasas crudas y estandarizadas por edad, pendientes de regresión con intervalos de confianza a $95 \%$, valor $p$ y coeficiente de determinación; porcentaje de cambio 80/97 e incremento medio anual (media geométrica), de 1980 a 1997. El consumo per capita de tabaco fue obtenido del INEGI ${ }^{17}$ (INEGI, 1994) y fue utilizado para estudiar la asociación entre el consumo previo de tabaco y las tasas crudas de mortalidad por cáncer de pulmón. El índice de marginación social para los 32 estados de la República fue estimado por Conapo ${ }^{18}$ (1998) utilizando diversos indicadores sociales recolectados en 1995 y correlacionados con las tasas de mortalidad por cáncer de pulmón, por entidad federativa. La información fue analizada con el paquete estadístico STATA versión 6.0 y SPSS, versión 10.

\section{Resultados}

La razón de género para la mortalidad por cáncer de la cavidad oral/laringe fue alrededor de 2.10:1.00 (hombre/mujer). La excepción fue el cáncer de laringe con una razón de 4.21:1.00; pulmón, $\not \subset 2.00$ h: 1.00 m; esófago $\not \subset 2.00 \mathrm{~h}: 1.00 \mathrm{~m}$; cavidad oral/faringe $\not \subset 2.00$ h: $1.00 \mathrm{~m}$; cáncer de laringe $=4.21 \mathrm{~h}: 1.00 \mathrm{~m}$.

La proporción relativa estimada, usando la mortalidad total por cáncer maligno de 1980 a 1997, fue mayor para cáncer del pulmón con $12.31 \%$, seguido de laringe $1.71 \%$, esófago $1.55 \%$ y cavidad oral/faringe, $1.49 \%$.

Las tasas crudas de mortalidad por cáncer de pulmón para todas las edades de hombres fluctuaron entre $5.52(1980)$ y 9.05 (1997) casos por $10^{5}$ hombres $p=0.1962$, IC95\% 0.1619, 0.2305; $p=0.000)$; el coeficiente de determinación (CD) fue $r^{2}=0.902$; el porcentaje de cambio $1980 / 1997,63.95 \%$ y el incremento medio anual para el periodo, de $2.95 \%$.

En el grupo de 35-64 años de edad, las tasas fluctuaron de 11.74 en 1980 a 11.71 en 1997, mostrando una elevada meseta en los años medios del intervalo. La mortalidad cruda de cáncer de pulmón en mujeres tuvo un aumento de 2.72 (1980) a 4.29 (1997) casos por 100000 en todas las edades de las mujeres. El coeficiente de regresión fue 0.0824 , (IC 95\% 0.0698, 0.0949; $p=0.000$ ), con un CD de $0.9236 ; 57.52 \%$ de cambio de 1980/1997, y un aumento de la media anual de $2.72 \%$. En el grupo de 35-64 años, se observó un aumento de 5.63 (1980) a 5.89 (1997) casos. El aumento de la media anual fue de $0.27 \%$ (figura $1 \mathrm{~A}$, cuadro I). Las tasas de mortalidad estandarizadas por edad fueron mayores para cáncer del pulmón en ambos géneros; los coeficientes de determinación fueron mayores que los de las tasas crudas, con algunas excepciones, para las mujeres (figura 2A, cuadro II). 

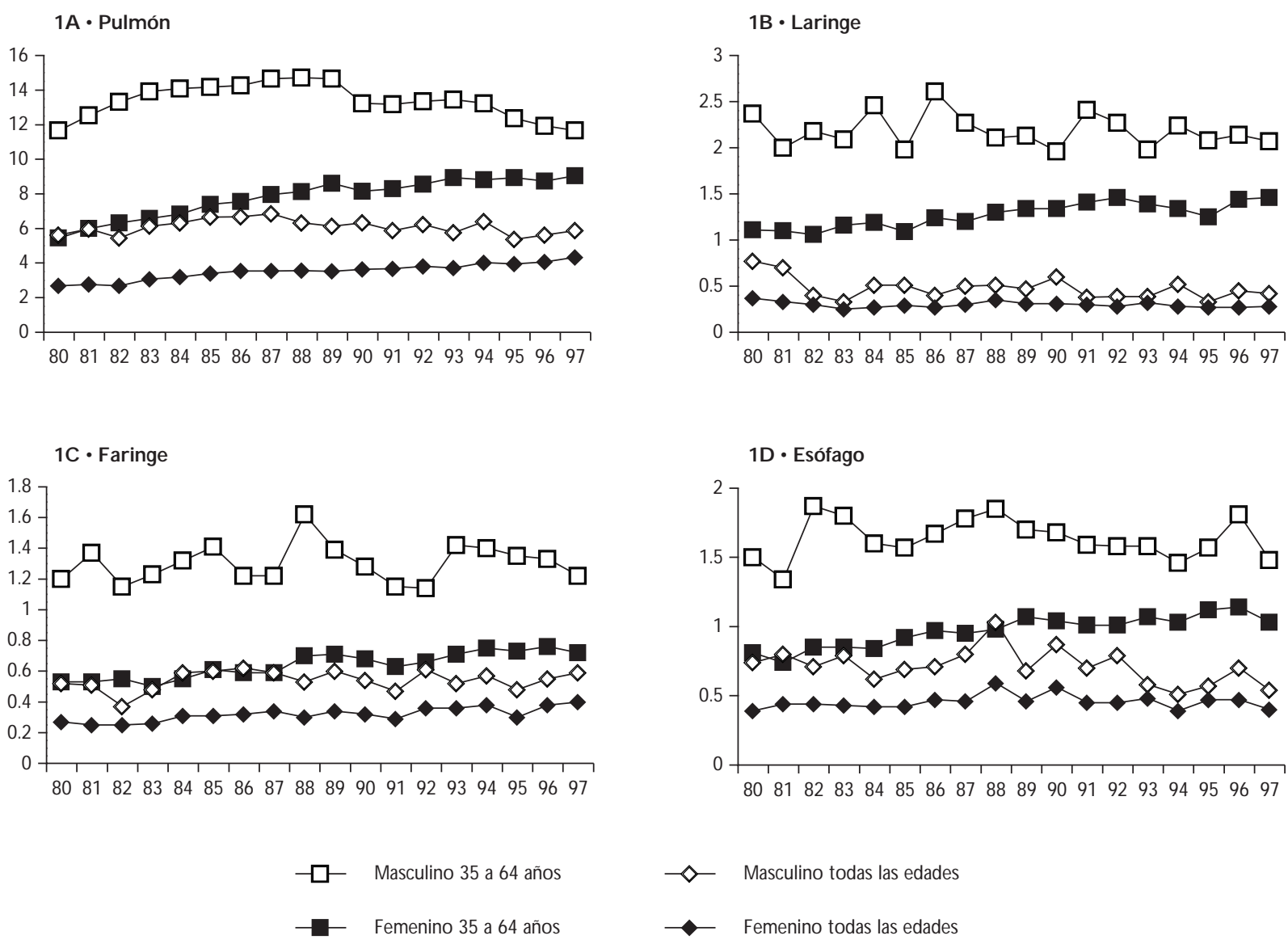

$\checkmark$ Masculino todas las edades

Figura 1.Tendencias de Las tASAS CRUdAs de MORTALIDAd POR CÁNCERES RELACIONADOS CON EL TABACO, POR GRUPO DE EDAD Y SEXO, en MéXICO (1980-1997)

Las tasas de mortalidad por cáncer de laringe en hombres de todas las edades de fue 1.12 (1980) y 1.45 (1997) casos en 100 000. El coeficiente de regresión fue 0.0207 (IC 95\% 0.0143, 0.0271; $p=0.000 ;$ CD 0.7466); hubo un porcentaje de cambio de 29.46 de 1980/1997 y el crecimiento de la media anual aumentó $2.06 \%$. En el grupo de 35-64 años, hubo una tendencia decreciente con un coeficiente de regresión de -0.0075 (IC 95\% $0.0252,0.0103 ; p=0.387 ; \mathrm{CD} 0.0471) ; \mathrm{y}$ un descenso de la media anual de $-0.82 \%$. Las tasas de mortalidad en todas las edades de los casos de las mujeres fue bajo, oscilando entre 0.36 (1980) y 0.29 (1997) por 100000. Este patrón también se observó en el grupo de 35-64 años. En ambos grupos de edad, los coeficientes de regresión fueron negativos. El cambio promedio 1980/1997 fue mayor para el grupo de 35-64 años de edad (-44.74\%) así como el descenso de la media anual $(-3.65 \%)$ (figura 1B, cuadro I). Cuando las tasas por edad de la mortalidad por cáncer de laringe se com- pararon, se observó mayor coeficiente beta de determinación en las tasas ajustadas (figura 2B, cuadro II).

El cáncer oral, de labios, lengua, paladar y faringe, como un todo, mostró una tasa de mortalidad de 0.53 (1980) y 0.72 (1997) por 100000 hombres. Este incremento representa un coeficiente de regresión de 0.0144 (IC 95\% 0.0110, 0.0179; $p=0.000$; CD 0.8290) con una variación promedio de $35.85 \%$ 1980/1997 y un aumento de la media anual de $1.82 \%$. La tasa de mortalidad en el grupo de edad de 35-64 años fue de 1.20 (1980) y 1.22 (1997) por 100000 hombres. En mujeres de todas las edades se observó un aumento de la media anual de $2.34 \%$ y una variación del promedio de $48.15 \%$ 1980/1997, siendo éste un aumento mayor que el que se observó en el grupo de 35-64 años (figura 1C, cuadro I). Previamente se describió un comportamiento semejante de los cánceres cuando se compararon las tasas crudas y ajustadas por edad de la mortalidad (figura 2C, cuadro II). 


\section{Cuadro I}

\section{Coeficientes de Regresión de las tASAS de mortalidad por CÁnCeres RelaCionados Con el tABACO, PORCENTAJe} de CAMbio en el Periodo e InCRemento medio anUAL POR GRUPOS de edAd y SEXo, MéXico (1980-1997)

\begin{tabular}{|c|c|c|c|c|c|c|c|}
\hline Sexo & Edad & Beta & $\begin{array}{l}\text { Intervalos de } \\
\text { confianza }\end{array}$ & Prob. & $\mathrm{R} 2$ & $\begin{array}{c}\text { Porcentaje de } \\
\text { cambio periodo* }\end{array}$ & $\begin{array}{l}\text { Incremento } \\
\text { medio anual }{ }^{\ddagger}\end{array}$ \\
\hline \multicolumn{8}{|l|}{ Masculino } \\
\hline \multirow[t]{2}{*}{ Pulmón } & $0-120$ & 0.1962 & $(0.1619,0.2305)$ & 0.00 & 0.90 & 63.95 & 2.95 \\
\hline & $35-64$ & -0.0533 & $(-0.141,0.0405)$ & 0.25 & 0.08 & -0.26 & -0.01 \\
\hline \multirow[t]{2}{*}{ Laringe } & $0-120$ & 0.0207 & $(0.0143,0.0271)$ & 0.00 & 0.75 & 29.46 & 2.06 \\
\hline & $35-64$ & -0.0075 & $(-0.0252,0.0103)$ & 0.39 & 0.05 & -13.08 & -0.82 \\
\hline \multirow[t]{2}{*}{ Faringe $^{\S}$} & $0-120$ & 0.0144 & $(0.0110,0.0179)$ & 0.00 & 0.83 & 35.85 & 1.82 \\
\hline & $35-64$ & 0.0008 & $(-0.0109,0.0125)$ & 0.88 & 0.00 & 1.67 & 0.10 \\
\hline \multirow[t]{2}{*}{ Esófago } & 0.120 & 0.0196 & $(0.0150,0.0243)$ & 0.00 & 0.84 & 28.40 & 1.48 \\
\hline & $35-64$ & -0.0019 & $(-0.0164,0.0126)$ & 0.79 & 0.00 & -0.67 & -0.04 \\
\hline
\end{tabular}

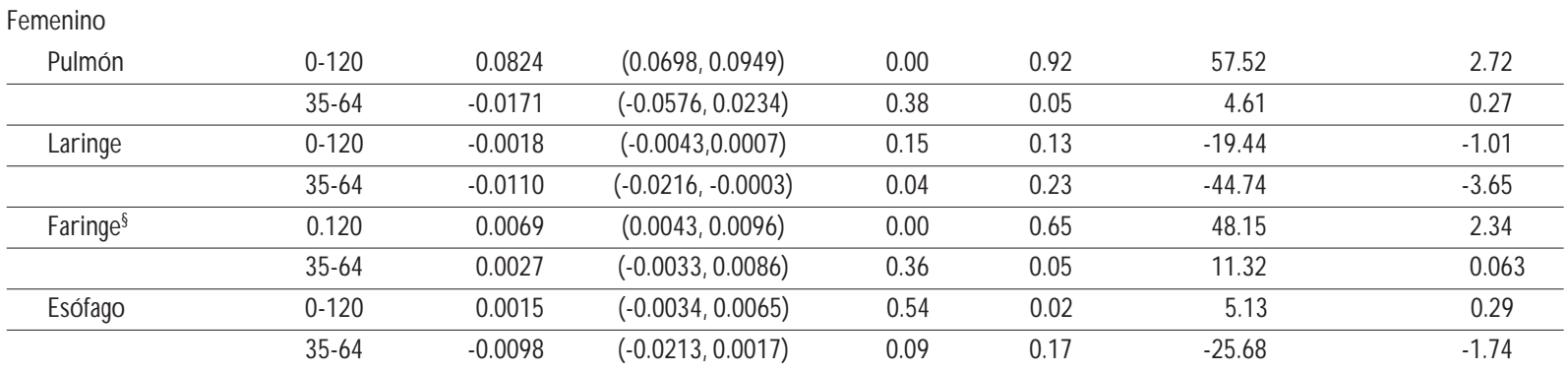

*1980 vs 1997

抽 1980 a 1997

§Incluye labio, lengua y paladar

El cáncer de esófago en los hombres de todas las edades mostró un aumento en las tasas de mortalidad de 0.81 casos (1980) a 1.04 (1997) por 100000 , con un coeficiente beta positivo de 0.0196 (IC 95\% 0.0150, 0.0243; $p=0.000$; CD 0.8354). La variación promedio 1980/1997 fue $28.40 \%$ y el aumento de la media anual, $1.48 \%$. Para el grupo de 35-64 años, se dio una pequeña reducción en la tasa de mortalidad de 1.49 (1980) a 1.48 casos (1997). En las mujeres se observó un comportamiento similar por grupos de edad, con un aumento discreto a todas las edades de 0.39 (1980) a 0.41 (1997) por 100 000. El grupo de 35-64 años mostró un coeficiente de regresión negativo; no obstante, no fue estadísticamente significativo (figura 1D, cuadro I). Cuando se comparan las tasas de mortalidad crudas y ajustadas por edad se observa un aumento en estas últimas (figura 2D, cuadro II).

Se obtuvieron correlaciones positivas bivariadas de Pearson, con coeficientes de 0.9 a 0.7 entre las tasas de mortalidad por cáncer de pulmón (1980-1997) para el grupo de 35-64 años, y el consumo de tabaco per capita, usando un periodo de tiempo de exposición de 27-30 años (cuadro III). Al comparar el consumo de tabaco per capita y las tasas de mortalidad por cáncer de pulmón se observa una pendiente con coeficiente de regresión de 0.910 (IC 95\% 1.097, 1.797; p=0.000; CD 0.827). Esta se obtuvo usando 28 años de tiempo de exposición. Este resultado podría interpretarse como que a mayor consumo de tabaco, mayores tasas de mortalidad por los años de exposición (figura 3). El grupo de todas las edades no mostró correlación entre el tiempo de exposición del consumo de tabaco per capita y las tasas de mortalidad por cáncer de pulmón crudas y ajustadas por edad. Asimismo, otros cánceres atribuibles al consumo de tabaco mostraron pequeña o ninguna correlación. Cuando las tasas de mortalidad crudas fueron correlacionadas con el índice de marginación social, se observó una asociación inversa; a mayor marginación por estado, las tasas de mortalidad fueron menores (coeficiente de regresión $=-0.510$, IC 95\%: $-0.170,-0.039 ; p=0.003$, CD 0.260). 
2A • Pulmón

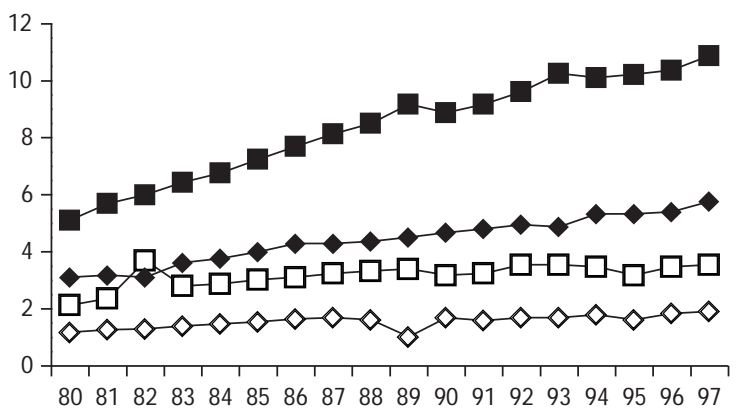

$2 C \cdot$ Laringe

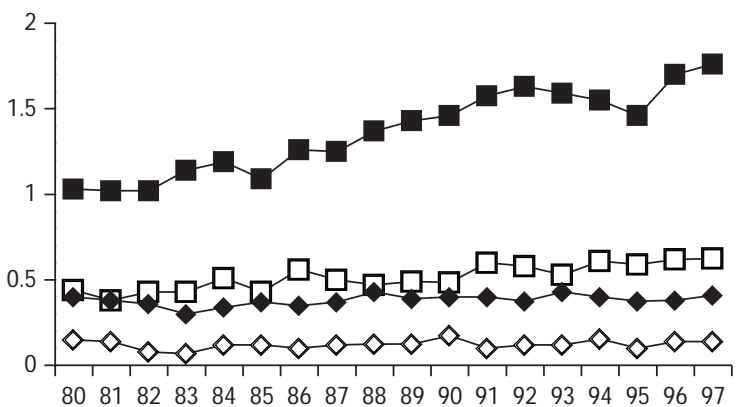

2B • Esófago

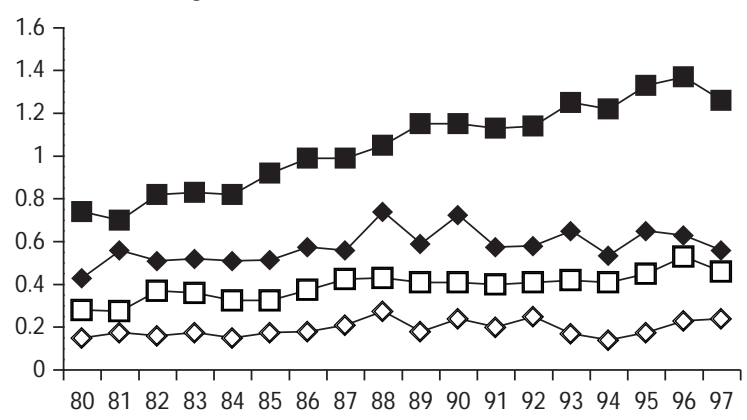

2D • Faringe

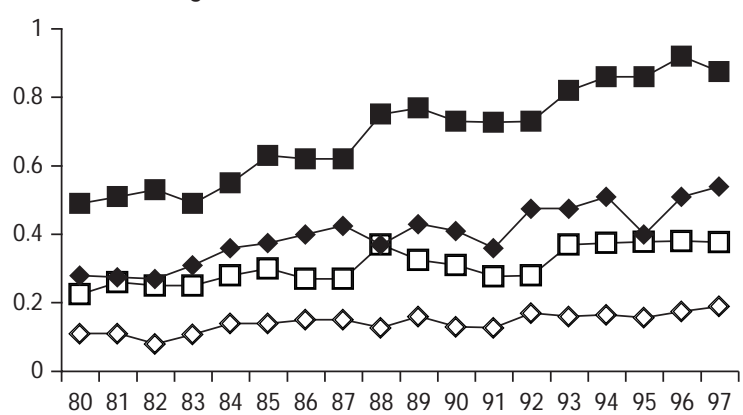

$\neg \square \quad$ Masculino 35 a 64 años

$\checkmark$ Masculino todas las edades

- Femenino 35 a 64 años

Femenino todas las edades

Figura 2.Tendencias de LAS TASAS De MORTALIDAd AJUSTADAS POR EDAD, POR GRUPOS DE EDAD Y SEXO, POR CÁNCERES relacionados con el tabaco en México (1980-1997)

\section{Discusión}

Desde 1979 ha mejorado considerablemente tanto la calidad del registro del cáncer, como la clasificación de los certificados de defunción en México. Actualmente se dispone de una base de datos con la mortalidad y otras 22 variables. Sin embargo, no hay un registro nacional de casos nuevos, por lo que no es posible identificar la incidencia, distinguiendo su efecto compensatorio sobre el análisis de la sobrevivencia y la mortalidad por cáncer atribuible al consumo de tabaco. La información ha alcanzado un nivel aceptable de estabilidad y actualización; se han colectado 18 puntos en tiempo, pero el registro tiene aún espacio para mejorar. El cáncer del pulmón es el más frecuente en el mundo, ${ }^{19}$ y sus tendencias en incidencia y mortalidad están aumentando, debido principalmente a tres factores: a) mayor acceso al diagnóstico sensible y específico y más precisión para identificar la causa de la muerte, particularmente en personas de la tercera edad. Consecuentemente, se reconoce como tal una porción significativa de este cáncer mortal; ;0,21 b); está en aumento el número absoluto de la población de adultos en el mundo con riesgo de desarrollar cáncer, y c) el incremento relevante en el número de personas que fuman tabaco está produciendo elevaciones significativas en la tasa de mortalidad por cáncer atribuible a este consumo.

En México, los determinantes más importantes para aumento en el registro de cánceres son los siguientes: mejor sensibilidad y especificidad del diagnóstico, cambios en la población y aumento en el consumo de tabaco. No obstante, otras variables asociadas con los estilos de vida como la urbanización, la actividad física, el consumo de carotenoides y otros compuestos dietéticos, la ingesta de alcohol etílico, podrían estar influyendo en las tasas de mortalidad. ${ }^{5,19}$ Más aún, la publicidad y la venta de tabaco en 


\section{Cuadro II \\ Coeficientes de Regresión de LAS tASAS de MORTALIDAd POR CÁnCERES ATRIBUIBLES AL tABACO, AJUSTADAS POR EDAD, PORCENTAJE DE CAMBIO EN EL PERIODO Y PORCENTAJE ANUAL DE CAMBIO POR GRUPOS DE EDAD Y SEXo, MÉXICO (1980-1997)}

$\begin{array}{lllll}\text { Sexo } & \text { Intervalo de } & \text { Porcentaje de } & \begin{array}{c}\text { Porcentaje de } \\ \text { cambio periodo* }\end{array} \text { cambio anual }\end{array}$

\begin{tabular}{|c|c|c|c|c|c|c|c|}
\hline \multicolumn{8}{|l|}{ Masculino } \\
\hline \multirow[t]{2}{*}{ Pulmón } & $0-120$ & 0.3310 & $0.3007,0.3613$ & 0.00 & 0.97 & 112.48 & 4.53 \\
\hline & $35-64$ & 0.0659 & $0.0467,0.0851$ & 0.00 & 0.77 & 61.19 & 4.53 \\
\hline \multirow[t]{2}{*}{ Laringe } & $0-120$ & 0.0437 & $0.0368,0.0507$ & 0.00 & 0.92 & 69.90 & 3.17 \\
\hline & $35-64$ & 0.0121 & $0.0081,0.0160$ & 0.00 & 0.72 & 40.91 & 2.04 \\
\hline \multirow[t]{2}{*}{ Faringe $^{\S}$} & $0-120$ & 0.0256 & $0.0219,0.0292$ & 0.00 & 74.00 & 3.31 & \\
\hline & $35-64$ & 0.008 & $0.0060,0.0115$ & 0.00 & 0.74 & 65.22 & 3.00 \\
\hline \multirow[t]{2}{*}{ Esófago } & $0-120$ & 0.0375 & $0.0327,0.0423$ & 0.00 & 0.95 & 68.91 & 3.13 \\
\hline & $35-64$ & 0.0099 & $0.0067,0.0131$ & 0.00 & 0.73 & 60.71 & 2.83 \\
\hline
\end{tabular}

\begin{tabular}{cccccccc}
$\begin{array}{c}\text { Femenino } \\
\text { Pulmón }\end{array}$ & $0-120$ & 0.1516 & $0.1382,0.1650$ & 0.00 & 0.97 & 87.10 & 3.77 \\
\hline & $35-64$ & 0.0307 & $0.0212,0.0401$ & 0.00 & 0.75 & 58.33 & 2.74 \\
\hline Laringe & $0-120$ & 0.0022 & $-0.0008,0.0053$ & 0.14 & 0.13 & -2.44 & -0.23 \\
\hline & $35-64$ & 0.0002 & $-0.0023,0.0027)$ & 0.86 & 0.00 & -17.65 & -1.82 \\
\hline Faringe§ $^{\text {Esófago }}$ & $0-120$ & 0.0132 & $0.0097,0.0167$ & 0.00 & 0.80 & 80.00 & 3.52 \\
\hline & $35-64$ & 0.0040 & $0.0025,0.0056$ & 0.00 & 0.66 & 72.73 & 3.27 \\
\hline & $0-120$ & 0.0081 & $0.0017,0.0146$ & 0.02 & 0.31 & 27.91 & 1.46 \\
\hline
\end{tabular}

*1980 vs 97

林 1980 a 1997

\$ncluyendo labios, paladar y lengua

los países en desarrollo están menos reguladas que en los países desarrollados económicamente lo cual podría contribuir a su consumo creciente..$^{22}$

La correlación entre el índice de marginación social y las tasas de mortalidad por cáncer del pulmón por entidad federativa, refleja un patrón diferente del actual en países en desarrollo, en los cuales la mayor marginación está asociada con mayores tasas de mortalidad. ${ }^{9}$ En México, la correlación es inversa; los estados con mayores tasas de mortalidad por cáncer del pulmón tienen menores índices de marginación. Esto probablemente se debe al mayor acceso a bienes y tabaco, mayor ingreso económico y diferencias en estilo de vida entre las comunidades con diferentes niveles en su desarrollo social y económico. Por lo mismo, el aumento de la prevalencia de cáncer del pulmón asociada a la pobreza, descrito en algunos países, no se observa entre las regiones con menor nivel de desarrollo económico en México. ${ }^{22,23}$

Los cánceres de labios, cavidad oral, laringe y esófago comparten características comunes. Aun- que están asociados con el consumo de tabaco, el alcohol etílico y algunas dietas también se asocian con los mismos. ${ }^{24}$

El análisis de la tasa de mortalidad por género es un instrumento estadístico útil no sólo desde el punto de vista de la salud colectiva, sino también como un marcador cuantitativo de la importancia relativa de la enfermedad por género. Provee información etiológica confiable que potencialmente puede derivarse de sus resultados..$^{25}$ En este estudio las tendencias de mortalidad se analizaron por edad y género. Se estimaron los datos crudos y ajustados por edad en el periodo de estudio. Las tasas crudas y ajustadas por edad de la mortalidad por cáncer fueron similares en intensidad y dirección, pero algunos subgrupos mostraron diferencias mayores. Como esperábamos, se encontró un aumento en la tendencia de la tasa de mortalidad entre los hombres en virtud de que los factores de riesgo, y particularmente el consumo de tabaco, eran mayores. Durante la década de los 50, la comercialización de los 
Cuadro III

Coeficientes de correlación bivariados entre el CONSUMO PER CAPITA PREVIO DE TABACO (30 A 27 AÑOS ANTES) Y LA TASA CRUDA DE MORTALIDAD POR CÁNCER PULMONAR EN MÉXICO (1980-1997)*

\begin{tabular}{lccc}
$\begin{array}{l}\text { Consumo per capita } \\
\text { De tabaco }\end{array}$ & \multicolumn{3}{c}{ Tasa de cáncer pulmonar } \\
\cline { 2 - 4 } 30 años & Total & Masculino & Femenino \\
\hline 29 años & $0.753^{\ddagger}$ & $0.729^{\ddagger}$ & $0.604^{\ddagger}$ \\
\hline 28 años & $0.891^{\ddagger}$ & $0.838^{\ddagger}$ & $0.727^{\ddagger}$ \\
\hline 27 años & $0.910^{\ddagger}$ & $0.801^{\ddagger}$ & $0.822^{\ddagger}$ \\
* G rupo de edad: 35 a 64 años \\
₹ Coeficiente de correlación significativo a nivel de 0.01 (2-colas)
\end{tabular}

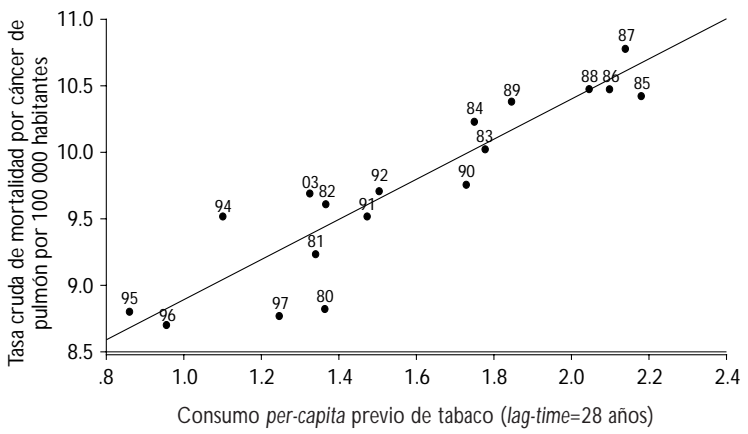

Figura 3. Análisis de regresión lineal de las tASAS CRUDAS DE MORTALIDAD POR CÁNCER DE PULMÓN Y CONSUMO PREVIO DE TABACO EN MEXICANOS DE 35 A 64 AÑOS DE EDAD (1980-1997)

cigarrillos con filtro aumentó dramáticamente el consumo de tabaco entre las mujeres. Este hecho podría explicar parcialmente por qué la tasa de mortalidad se está uniformando con la de los hombres. ${ }^{26}$ Algunos autores sugieren que si las mujeres fumaran tabaco tanto como los hombres, el número de muertes por cáncer sería semejante. ${ }^{5}$ Otros autores tienen la hipótesis de que las mujeres son más susceptibles que los hombres ${ }^{27}$ a los efectos dañinos del tabaco. En México, necesitamos estudiar esta hipótesis en el análisis de la tendencia de mortalidad secular.

Cuando se analizó la tendencia de las tasas crudas y ajustadas por edad de la mortalidad por cáncer de pulmón se encontró una reducción en el grupo de hombres de 35 a 65 años. Por lo tanto, es posible inferir que el aumento en la mortalidad se debió principalmente a expensas del cáncer en la población mayor de
65 años de edad. Las mujeres mostraron incremento de las tasas de mortalidad tanto en el grupo de 35 a 65 años de edad como en el grupo con todas las edades.

Aunque el cáncer de pulmón no sólo está asociado con el consumo previo, sino también con el tiempo, patrón y frecuencia de exposición, ${ }^{28}$ la correlación entre el consumo previo per capita de tabaco y las tasas crudas de mortalidad por cáncer de pulmón de 1980 a 1997 fue significativa en el grupo de 35 a 64 años. Pero estuvo ausente en el grupo de todas las edades. Esta correlación bajó cuando el tiempo de exposición aumentó o descendió, lo cual sugiere un periodo de incubación de cerca de 28 a 30 años. Otros cánceres atribuibles al tabaco mostraron poca o ninguna correlación significativa con el consumo previo de tabaco per capita. Esta información debe ser interpretada con cautela en virtud de que no es posible controlar otras variables relacionadas, ni incluir otros determinantes relevantes.

México ha estado mostrando un aumento importante de las ECNTs durante las tres últimas décadas. El cáncer atribuible al tabaco es una preocupación de salud pública, no solamente porque el pronóstico es pobre, sino porque también está aumentando dramáticamente el costo de la atención médica, y puede ser prevenido por medio de la educación para la salud, el aumento de los impuestos a los productos de tabaco y la regulación de la publicidad.

Los países en desarrollo deben realizar más investigación epidemiológica en relación con tendencias, proyecciones, muertes prematuras, magnitud y otros indicadores del cáncer atribuible al consumo de tabaco y la adicción a la nicotina. La generación del conocimiento sobre las complejas relaciones entre el tabaco y el cáncer en esta etapa de transición epidemiológica, y sobre los determinantes del problema, ayudará indudablemente a desarrollar programas de prevención eficaces y permanentes.

\section{Referencias}

1. Bo badilla J, Frenk J, Lozano R, Frejka,T, Stern C. The epidemiologic transition and health priorities. En:Jamison D, ed. Disease control priorities in developing countries. N ueva York (N Y): O xford University Press, 1993. 2. 0 mran AR.The epidemiologic transition theory.A preliminary update. J Trop Pediatr 1983;29:305-316.

3. 0 mran A R. The epidemiologic transition. A theory of the epidemiology of population change. Milbank Memorial Fund Q 1971;49:509-538.

4. LaVecchia C, Levi F, Lucchini F, N egri E, Boyle P.Trends in cancer mortality in the USSR, 1965-1990. Int J Cancer 1994;56:31-39.

5.W orld Cancer Research Fund. W CRF. Food, nutrition and the prevention of cancer:A global perspective.W ashington, D.C.:A merican Institute of Cancer, 1997:670. 
6. Prabhat J. Curbing the Epidemic. Governments and the economics of to bacco control.W ashington, D.C.:The International Bank for Reconstruction and D evelopment/The World Bank, 1999:122.

7. D oll R, Hill A. Smoking and carcinoma of the lung: Preliminary report. BMJ 1950;2:739-748.

8. Levin M. G oldstein H, Gerhardt P. C ancer and tobacco smoking: Preliminary report. JAMA 1950;143:336-338.

9. W ynder E, Goodman M. Smoking and lung cancer: Some unresolved issues. Epidemiol Rev 1983:5:177-207.

10.W ynder E, Graham E.Tobacco smoking as a possible ethiological factor in bronchiogenic carcinoma. JAMA 1950;143:329-333.

11. Boyle P. Cancer, cigarette smoking and premature death in Europe:A review including the Recommendations of European $C$ ancer Experts C onsensus Meeting, Helsinki, 0 ctober 1996. Lung C ancer 1997;17:1-60.

12. Lazcano PEC, Tovar GV, Meneses GF, Rascón PRA, Hernández AM. Trends of lung cancer mortality in Mexico. Arch Med Res, 1997;28: 565-570.

13. Chen ZM, X U Z, C ollins R, Li W X, Peto R. Early health effects of the emerging tobacco epidemic in China.A 16-year prospective study. JAMA 1997;278:1500-1504.

14. Firat D. Tobacco and cancer in Turkey.J Environ Pathol Toxicol 0 ncol 1996;15:155-160.

15. Lam TH, HeY, Li LS, Li LS, He SF, Liang BQ . Mortality attributable to cigarette smoking in $\mathrm{China}$ [see comments] [published erratum appears in JAMA 1998 May 6;279(17):1350]. JAMA 1997;278:1505-1508.

16. Instituto N acional de Estadística Geografía e Informática. Estadísticas Históricas de México. A guascalientes, Ags.: IN EGI, 1994.

17. O rganización Panamericana de la Salud. C lasificación Internacional de Enfermedades, IX revisión. W ashington, DC: O PS, 1978.
18. Consejo N acional de Población. Indices de marginación, 1995. México, D.F.: CONAPO, 1998.

19. Buiatti E, G eddes M,Arniani S. Epidemiology of lung cancer.A nnali dell Istituto Superiore di Sanita 1996;32:133-144.

20. D oll R, Peto R. The causes of cancer: Q uantitative estimates of avoidable risks of cancer in the U nites States to day.J N atl C ancer Inst 1981;66: 1191-1308.

21.W aterhouse J, Muir C , Powell J.C ancer incidence in five continents, Vol. III, Lyon (Fracia): International A gency for Research on C ancer, 1976.

22. Tomatis L. Poverty and cancer. Lyon (Francia): IARC Scientific Publications, 1997;138:25-39.

23. Tomatis L. Socioeconomic factors and human cancer. Int J Cancer 1995;62:121-125.

24. International A gency for Research on Cancer. Tobacco habits other than smoking: Betel-quid and areca-nut chewing and some related nitrosamines. Lyon, Francia: IARC, 1985.

25. Levi F, LaVecchia C, Lucchini F, N egri E.Trends in cancer mortality sex ratios in Europe, 1950-1989. World Health Stat Q 1992;45:117-123.

26. Britton GA.A review of women and tobacco: $\mathrm{H}$ ave we come such a long way? J O bstet Gynecol N eonatal N urs 1998;27:241-249.

27. Prescott $E, O$ sler $M$, Andersen $P K$, H ein $H O$, Borch-Johnsen $K$, Lange $P$, Schnohr P,Vestbo J. Mortality in women and men in relation to smoking. Int J Epidemiol 1998;27:27-32.

28. Nyberg F, A grenius V, Svartengren K, Svensson C, Pershagen G. Environmental tobacco smoke and lung cancer in nonsmokers: $D$ oes time since exposure play a role? Epidemiology 1998:9:301-308. 